\title{
El cuerpo más allá del organismo: el estatus del cuerpo en el psicoanálisis lacaniano*
}

\author{
The body beyond the organism. \\ The status of the body \\ in Lacanian psychoanalysis
}

\author{
FRANCISCO CONDE SOTO \\ Universidad de Santiago de Compostela
}

Recibido: 17/03/16 Aceptado:10/10/16

\begin{abstract}
RESUMEN
Este estudio presenta las reflexiones sobre el cuerpo del psicoanalista francés Jacques Lacan en diferentes momentos de su enseñanza. En una primera parte, se atiende al cuerpo según las tres categorías o registros de Lacan: imaginario (el cuerpo como imagen), simbólico (el cuerpo como deseo) y real (el cuerpo como goce imposible). En segundo lugar, se analiza la perspectiva del último Lacan, donde el cuerpo es pensado como aquello que ofrece consistencia al sujeto. El objetivo principal del estudio es demostrar que un eje común a todos estos enfoques es que el psicoanálisis lacaniano sitúa el cuerpo más allá del organismo.
\end{abstract}

PALABRAS CLAVE

CUERPO, DESEO, GOCE, LACAN, SUJETO

\footnotetext{
* Este artículo ha sido realizado en el marco del proyecto de investigación "Sufrimiento social y condición de víctima: dimensiones epistémicas, sociales, políticas y estéticas", financiado por el Programa Estatal de Fomento de la Investigación Científica y Técnica de Excelencia (FFI2015-69733-P).
}

(C) Contrastes. Revista Internacional de Filosofía, vol. XXII-N²2 (2017), pp. 7-22. ISSN: 1136-4076

Departamento de Filosofía, Universidad de Málaga, Facultad de Filosofía y Letras Campus de Teatinos, E-29071 Málaga (España) 


\begin{abstract}
This study presents the reflections on the body of the French psychoanalyst Jacques Lacan at different times of his teaching. In the first part, it meets the body according Lacan's three categories: imaginary (the body as an image), symbolic (the body as desire) and real (the body as an impossible enjoyment). Secondly, according to the perspective of the last Lacan, where the body is thought of as which provides consistency to the subject. The main objective of the study is to demonstrate that a common axis to all these approaches is that Lacanian psychoanalysis puts the body beyond the body.
\end{abstract}

KEYWORDS

BODY, DESIRE, ENJOYMENT, LACAN, SUBJECT

\title{
INTRODUCCIÓN
}

EL CUERPO Y SU RELACIÓN CON EL SUJETO ha sido y sigue siendo objeto de estudio preferente en el campo de la filosofía contemporánea continental. ${ }^{1} \mathrm{Mu}$ chas de estas aportaciones nacen a partir de o en diálogo con el psicoanálisis, en especial con la relectura posestructuralista del psicoanálisis freudiano que lleva a cabo el psicoanalista francés Jacques Lacan. La práctica psicoanalítica se enfrenta a sujetos que sufren y aborda este sufrimiento desde la palabra. Se trata de cuerpos sufrientes que hablan por medio de los malestares y síntomas que los aquejan siendo el objetivo del dispositivo psicoanalítico conseguir que estos malestares sean dichos y neutralizar así la carga de padecimiento que conllevan. Aunque los desarrollos de la teoría psicoanalítica apuntan y se reducen al papel de herramientas con vocación de servir a la práctica clínica, los hallazgos teóricos que Jacques Lacan formula a partir del discurso del sujeto que pasa por la experiencia psicoanalítica pueden ser generalizados para construir una teoría general de una subjetividad corporeizada. ${ }^{2}$ Ciertamente el psicoanálisis freudiano situaba ya al cuerpo como el elemento nuclear de la

1 En una primera lista confeccionada a salto de mata tendrían que figurar las aportaciones fundamentales de: Gilles Deleuze y Félix Guattari, especialmente su Mil mesetas. Capitalismo y esquizofrenia II (1981), Pierre Bordieu en «Notas provisionales sobre la percepción social del cuerpo» (1986), Jean Luc Nancy en Corpus (1992), Judith Butler en Cuerpos que importan (1993), Rosi Braidotti en Sujetos nomádicos (1994), Peter Sloterdijk en Esferas (1998), Jacques Derrida en El animal que luego estoy si(gui)endo (2006) o David Le Breton en Adiós al cuerpo (2013).

2 Cierto que de una naturaleza muy diferente al del enfoque de la «embodied mind» que ha irrumpido en los últimos diez años en el campo de la filosofía de la mente. Este enfoque sostiene reclamándose heredero de la tradición fenomenológica que el cuerpo «constriñe, distribuye o regula» las operaciones mentales (véase Wilson\&Foglia, 2015), llevando a cabo en líneas generales una especie de mentalización del cuerpo. El psicoanálisis y en general la filosofía continental propondrían más bien una corporeización de la mente, es decir, una explicación causal de ciertos comportamientos conscientes a partir del cuerpo. 
identidad del sujeto. Si bien el inconsciente es pensado en un primer momento principalmente como una parte de la psique situada en la constelación inconsciente, preconsciente y conciencia, la introducción de la segunda tópica-yo, ello y superyó- deja paso a un inconsciente que opera desde el ello y que es pensado por Freud como instinto -en una mala traducción del término alemán Trieb- y como pulsión -en una mejor traducción. Se entiende, así, que Freud resuma la operación psicoanalítica en la frase «Donde el ello era, debo advenir yo». ${ }^{3}$ Pero es Lacan quien lleva al extremo esta idea de que el inconsciente del sujeto no es ninguna entidad oscura sino que muy precisamente se trata de sucesos que tienen lugar en su cuerpo. Para entender el sentido y el peso de esta afirmación se hace necesario realizar un recorrido por los vericuetos de la teorización lacaniana del cuerpo. En primer lugar, intentando sistematizar los acercamientos que Lacan lleva a cabo en las tres épocas de su enseñanza, en cada una de las cuales prima uno de los tres registros, por supuesto sin borrar el papel de los otros dos. Y finalmente, atendiendo a eso que el principal intérprete de la obra de Lacan ha dado en llamar el 'últimísimo Lacan', donde se produce una cierta ruptura con los planteamientos anteriores y en el contexto de un vacimiento teórico del corpus conceptual del psicoanálisis el cuerpo adviene al lugar central. ${ }^{4}$

\section{EL CUERPO IMAGINARIO}

Lacan repiensa el psicoanálisis de Freud proponiendo tres registros -lo imaginario, lo simbólico y lo real- que, sin dejar de coexistir a lo largo de toda su enseñanza, ocupan una posición más o menos destacada dependiendo de la época. Durante una época primera Lacan piensa que el psicoanálisis consiste en la localización y toma de conciencia de los complejos o «imagos» del sujeto, tal y como formula en un texto de $1937 .{ }^{5}$ En esta época es precisamente la imagen entendida como una cierta representación o constructo del sujeto, aunque no del orden de la ficción, sino con claros efectos en realidad, la que ocupa el lugar central. Partiendo de un experimento de 1931 del psicólogo francés Henri Wallon, Lacan recuerda que el infante gana una vivencia de su cuerpo como algo unitario a partir de la percepción de la unidad que muestra la imagen de su propio cuerpo en un espejo. La imagen le permite así constituir su propia identidad, una identidad que justo un instante antes no existía. ${ }^{6}$ El yo gana su

3 «Wo Es War, soll Ich Werden» (Freud 1933, p. 86).

4 Este es precisamente el título de un curso sobre Lacan impartido por Jacques-Alain Miller en el curso académico 2006/2007 (Miller 2013).

5 «Estos efectos presentan caracteres tan distintos y contingentes que obligan a considerar como elemento fundamental del complejo esta entidad paradójica: una representación inconsciente, designada con el nombre de imago» (Lacan 1937, p. 29).

6 El texto original de la exposición hecha en el XVI Congreso psicoanalítico Internacional de Marienbad (2-8 de agosto de 1936) fue extraviado. Una exposición detallada de la teoría del 
identidad gracias a una identificación con su propia imagen, de ahí que sea este un ejemplo óptimo para entender que la categoría de lo imaginario no es algo líquido o poco consistente, sino muy al contrario aquello que le presta consistencia al cuerpo.

Para Lacan, antes de que tenga lugar este proceso el sujeto vive en un cuerpo fragmentado, un amasijo de órganos sin orden, descoordinados unos en relación a los otros, tal y como se muestra en algunos sueños o en algunas patologías esquizofrénicas, donde los órganos funcionan solos o donde aparecen órganos fantásticos que se viven como partes totalmente ajenas al propio cuerpo. ${ }^{7}$ Es la percepción de su imagen en el espejo la que le permite al sujeto salir de esta situación. Esta unidad, percibida en un primer instante en la imagen exterior, actúa al modo de una anticipación de la unidad con la que el sujeto podrá sentirse en un cuerpo unificado a continuación. El sujeto provoca su propio proceso de maduración mediante una especie de espejismo en el que la «ficción» de la unidad ahí fuera acaba por tener efectos de configuración reales sobre como se vive a sí mismo ahí dentro. El estadio del espejo, fase que estaría en la historia de todos los sujetos, consiste en esta transformación producida en el sujeto cuando asume su imagen, que le permite así la conquista de la unidad de sí mismo. Por otra parte, y en diálogo con la teoría de la Gestalt, para Lacan el cuerpo del sujeto se convertiría así en la unidad, forma y figura primeras que, aplicada a la multiplicidad de sensaciones del mundo exterior, permite que estos sean estructurados y ordenados como objetos. En definitiva, durante esta primera época el cuerpo será para el psicoanálisis de Lacan ante todo una imagen unitaria, no en el sentido de mera representación sino pensada como principio ordenador y estructurante.

\section{El CUERPO SIMBÓLICO.}

La peculiar adaptación de la concepción del signo de Saussure que lleva a cabo Lacan consiste principalmente en negar la primacía del significado (representación o concepto mental) sobre el significante (representante material) para pasar a sostener que el significado o está ausente o como mínimo siempre desplazado. Para distinguir su concepción del lenguaje de la de Saussure

espejo es recogida en el texto «Nota sobre la exposición de Daniel Lagache» (Lacan 1966, pp. 647-684).

7 En este sentido, sobre el jurista alemán Schreber, caso de esquizofrenia paranoide sostenía Freud: «Sostiene haber experimentado en los primeros años de su enfermedad destrucciones en diversos órganos de su cuerpo, que la cualquier otro hombre le habrían provocado indefectiblemente la muerte desde mucho tiempo atrás, pero él ha vivido un largo período sin estómago, sin intestinos, sin pulmones casi, con el esófago desgarrado, sin vejiga, con las costillas rotas, muchas veces se ha comido parte de su laringe al tragar, etc.» (Freud 1911, p. 17). 
substituye el término signo por el del significante y se refiere al conjunto de los significantes como el tesoro de los significantes o como el Otro simbólico.

El registro de lo simbólico es el registro al que pertenece todo aquello capaz de tener efectos de significación. No solo el lenguaje y la palabra, sino cualquier cosa capaz de significar pueden ser considerados como significantes. Para Lacan tiene el estatuto de dogma la creencia de que los hechos para ser tales necesitan ser dichos y de que no hay abolutamente nada en eso que consideramos la realidad que no sea sostenido por el hecho de poder ser expresado en significantes. ${ }^{8}$ La materia de la que está hecha la realidad para el psicoanálisis son los significantes que la ordenan. La realidad en sentido común no es más que aquello que los significantes consiguen articular a través de las remisiones de unos a otros.

El descubrimiento de este Otro no es exclusivamente teórico, sino que tiene también que ver con la experiencia psicoanalítica. Con el paso del tiempo Lacan descubre que la supuesta relación intersubjetiva entre paciente y psicoanalista, o entre el paciente y aquellas personas de las que habla en un psicoanálisis, consiste más bien en una relación entre el paciente y el conjunto de sus dichos, es decir, el conjunto de los enunciados que emite. A este conjunto lo denominará Otro, escrito con $\mathrm{O}$ mayúscula, inaugurando así la época más estructuralista y de predominio del registro de lo simbólico en Lacan. ${ }^{9}$

En realidad, es a partir de Freud como Lacan introduce la idea de que el inconsciente solo puede ser comprendido a partir del registro de lo simbólico. Si el psicoanálisis nacía con Freud como el intento de curar las parálisis histéricas para las que no se encontraba ninguna explicación fisiológica, es porque la histeria le permitió al vienés descubrir, en primer lugar, que hay malestares que se sitúan en el cuerpo pero que no tienen un origen fisiológico-orgánico; y en segundo lugar, que la estructura anatómica de los nervios y de los músculos es diferente de la estructura del cuerpo, de sus zonas, órganos o funciones. Dicho de otro modo, la histeria tiene que ver con un cuerpo que ya ha pasado por el filtro del lenguaje, puesto que como acabará formulando Lacan en 1970 en Radiofonía, es el lenguaje el que nos atribuye órganos, el que organiza nuestro cuerpo. $^{10}$

8 «Todo lo que hay en el mundo solo se vuelve propiamente un hecho si se articula con el significante» (Lacan 1968-69, p. 61).

9 Esta relación entre sujeto y gran Otro el psicoanalista es formalizada en un esquema que se conoce como Esquema L (Lacan 1952-53, p. 365) donde al eje especular/imaginario se superpone el eje de la palabra/simbólico, que es aquel donde Lacan pasará a situar el inconsciente.

10 «Vuelvo, en primer lugar, al cuerpo de lo simbólico que de ningún modo hay que entender como metáfora. A prueba de que nada sino él aísla el cuerpo tomado en sentido intuitivo, sea aquel en el que el ser que en él se sostiene no sabe que es el lenguaje que se lo adjudica, hasta el punto de que no se constituiría si no pudiera hablar» (Lacan 1974, p. 18). 
El cuerpo para el psicoanálisis ya no será el cuerpo de la anatomía y ahora tampoco el cuerpo de la imagen, sino el resultado de la organización que sobre el organismo pone en marcha el lenguaje. En Freud se hacía patente que el cuerpo va poco a poco obedeciendo a las regulaciones simbólicas de los sujetos que lo rodean, puesto que sus necesidades se ordenan según las demandas de la madre y el padre. Este proceso progresivo de significantización del cuerpo hace que pase de ser una imagen a transformarse en una totalidad de elementos discretos que se articulan gracias al lenguaje.

El cuerpo habitualmente pensado como una cosa sólida, de carne y hueso, es aquí más bien un cuerpo hablado, un cuerpo del que se dicen cosas, un cuerpo atravesado por los dichos y los deseos de las personas cercanas que impactan sobre él y lo recortan, lo tallan como trazos de un cincel, un cuerpo conversado cuya estructura puede ser recuperada tan solo a partir de los relatos del sujeto sobre él. Si no fuese gracias a los significantes que se inscriben sobre él, el cuerpo no sería en realidad más que una especie de vacío intangible.

Así, la frase de Freud «la anatomía es el destino» ${ }^{11}$ será aceptada por Lacan siempre y cuando se recuerde que la anatomía debe ser pensada ante todo como una operación -nunca mejor dicho- que el lenguaje lleva a cabo sobre el cuerpo a consecuencia de la cual este es organizado en unos peculiares objetos que ya no coinciden con lo orgánico y que Lacan acabará llamando objetos-causa del deseo u objetos pequeños a. Pensando el deseo Lacan llega a la conclusión de que el verdadero objeto del deseo no está realmente ahí delante, como siendo apuntado intencionalmente, sino más bien detrás, en tanto que objeto que causa ese deseo. ${ }^{12}$ La relación con su cuerpo de un sujeto es para el psicoanálisis, por lo tanto, una relación con estos peculiares objetos. Más allá de mostrarse como encarnado en determinado objeto particular exterior, el cuerpo y los objetos en los que se organiza no pertenecen al registro imaginario -no puede reflejarse en un espejo, no ofrecería propiamente hablando ninguna imagen situado delante de un espejo- sino al registro simbólico.

\section{EL CUERPO REAL: EL CUERPO GOZADO.}

En realidad muy pronto en la enseñanza de Lacan aparece la idea de que al Otro simbólico, es decir, al lenguaje, le falta algo, hecho que lo lleva a representar

11 Freud 1925, p. 185.

12 «Para fijar nuestro punto de mira, diré que el objeto a no debe situarse en nada que sea análogo a la intencionalidad de una noesis. en la intencionalidad del deseo, que debe distinguirse de aquélla, este objeto debe concebirse como la causa del deseo. Para retomar mi metáfora de hace un momento, el objeto está detrás del deseo» (Lacan 1962-63, p. 114). 
a este Otro con una A mayúscula atravesado diagonalmente por una barra. ${ }^{13}$ El Otro no es perfecto sino que carece de algo. Y esto de lo que carece es algo que no puede ser dicho ni simbolizado ni representado y que Lacan denomina técnicamente como 'real', un peculiar real que es al mismo tiempo imposible. ${ }^{14}$ Este concepto explica también el hecho de que después de un largo psicoanálisis queda siempre algo por decir y que rechaza entrar en el lenguaje. La cuestión es que esto imposible de simbolizar no será una mera nada sino que en su lugar Lacan pondrá algo, algo que dice obtener de una relectura de Freud.

Tradicionalmente Freud venía pensando la psique como una lucha entre los principios de placer y realidad: en virtud de la necesidad de adaptarse al mundo real el sujeto renuncia a la satisfacción inmediata de sus ansias de placer, consiguiendo así además acceder a placeres más duraderos. Ahora bien, como muy tarde en Más allá del principio del placer (1920) Freud renuncia definitivamente a explicar la conducta humana en base al principio del placer y afirma que aunque este principio pudiese conservar su validez en el ámbito de la conciencia, la conducta de los sujetos está condicionada desde su inconsciente por una compulsión a la repetición contraria al placer. Entre las diferentes objeciones que formula al principio del placer destaca la existencia de la compulsión a la repetición de escenas penosas en las neurosis traumáticas -muchos de los soldados regresados del frente de la I Guerra Mundial tenían repetida e insistentemente la misma pesadilla angustiante o no podían dejar de recordar una vivencia traumática-, pero sobre todo la resistencia a la curación y los numerosos retrocesos terapéuticos de sus pacientes. ${ }^{15}$ Es por ello por lo que formula que, si bien conscientemente los sujetos pueden perseguir el placer, a nivel inconsciente rige una cierta «pulsión de muerte» consistente en la tendencia innata de los sujetos a tender hacia la muerte y a una situación de

13 «Es lo que, en mis pequeñas fórmulas, les he llamado $\mathrm{S}(\mathrm{A} /)$, el significante de $\mathrm{A}$ tachado. Se trata con toda precisión de lo que acabo de definir como la función del significante falo, a saber, la de marcar lo que el Otro desea en cuanto marcado por el significante, es decir, tachado» (Lacan 1957-58, p. 375).

14 «Di esa fórmula casi algebraica, casi demasiado transparente, demasiado concreta; lo real o lo que es percibido como tal es lo que resiste absolutamente a la simbolización» (Lacan 1953-54, p. 110). «[...] como lo opuesto a lo posible es con toda certeza lo real, tendremos que definir lo real como lo imposible» (Lacan 1961, p. 164).

15 «En vista de estas observaciones relativas a la conducta durante la transferencia y al destino fatal de los seres humanos, osaremos suponer que en la vida anímica existe realmente una compulsión de repetición que se instaura más allá del principio de placer» (Freud 1920, p. 22). Los sueños traumáticos: «Nos proporcionan así una perspectiva sobre una función del aparato anímico, que sin contradecir al principio delplacer, es empero independiente de él y parece más originaria que el propósito de ganar placer y evitar displacer» (Freud 1920, p. 31). 
quietud previa a la vida. ${ }^{16}$ Freud termina su escrito con una pregunta acerca de cuál es la función de esta extraña compulsión de repetición.

Lacan desarrolla su concepto de goce para intentar responder a esta cuestión. De entrada, en El seminario 7: la ética del psicoanálisis introduce la idea de que cada sujeto está siempre en una peculiar relación con una cierta cosa que lo trasciende. «La cosa» [das Ding] es una noción deudora del nóumeno o cosaen-sí de Kant, pero sobre todo del texto heideggeriano de 1950 con ese mismo título. Heidegger introduce la noción de una cosa que no debe confundirse con el objeto, cuya esencia queda siempre más allá de todo aparecerse y que el ideal de dominación técnica de la sociedad contemporánea habría hecho olvidar. ${ }^{17}$ Lacan afirma que en realidad el sujeto pone en marcha una serie de defensas para mantener esta Cosa inquietante a una cierta distancia de manera que no lo invada: la belleza, la ley moral Kantiana y el deseo, entre otros, serían modos de dar vueltas en torno a un objeto imposible de soportar para el sujeto y con el que sostiene un juego de atracción-repulsión.

Poco a poco Lacan irá dándole ‘contenido' en su enseñanza a esta peculiar Cosa y será el goce lo que venga a su lugar. Mientras que el placer y el displacer se juegan de este lado de la barrera, más allá se produce una mezcla de ambos que, al mismo tiempo que dolorosa, provoca cierta satisfacción. De hecho, Lacan interpreta que el principio del placer freudiano tenía por único objetivo invitar al sujeto a quedarse más acá de este límite y mantenerlo alejado de su goce [jouissance ${ }^{18}$ ]. El goce queda del lado de la Cosa, fuera del sistema, fuera de lo simbolizable, sin que se pueda acceder a él de manera directa, ni siquiera mediante la transgresión, como sugería Lacan al recordar a Antígonaen el seminario VII, La ética del psicoanálisis, pero como negará diez años más tarde, al sostener que la relación con el goce es la relación con algo que se manifiesta tan solo al modo de un excedente. ${ }^{19}$ Con esta noción Lacan pretende explicar

16 «Una pulsión sería entonces un esfuerzo inherente a lo orgánico vivo, de reproducción de un estado anterior que lo vivo debió resignar bajo el influjo de fuerzas perturbadoras externas» (Freud 1920, p. 36). «Esa hipótesis deriva una pulsión de la necesidad de restablecer un estado anterior» (Freud 1920, p. 56).

17 «Algo autónomo puede convertirse en objeto si lo ponemos ante nosotros, ya sea en la percepción sensible inmediata, ya sea en el recuerdo que lo hace presente. Sin embargo, la cosidad de la cosa no descansa ni en el hecho de que sea un objeto representado (ante-puesto), ni en el hecho de que se puede determinar desde la objetualidad del objeto» (Heidegger 1950, p. 122).

18 «[...] está claro que la primera formulación del principio del placer como principio del displacer o de menor-padecer, entraña con toda seguridad un más allá, pero que está hecho justamente para mantenernos más acá de él. Su uso del bien se resume a que, en suma, éste nos mantiene alejados de nuestro goce» (Lacan 1959-60, p. 224).

19 «La relación al goce se acentúa de repente por esta función todavía virtual que se llama función del deseo. También es por esta razón por la que yo articulo como plus-de-gozar 
la resistencia a la curación de los sujetos y esa cierta atracción que provoca el sufrimiento en ellos, algo que dificulta enormemente la cura psicoanalítica. Se trata de una satisfacción en el dolor, de una satisfacción que procede de la satisfacción de la pulsión de muerte y que explica la resistencia de los síntomas del sujeto a desaparecer. El goce es aquella satisfacción inconsciente substitutiva, un placer que no puede ser sentido como tal, un satisfacerse en el displacer de los síntomas y el sufrimiento a ellos aparejado. Años más tarde, para explicar la consistencia del goce Lacan sostendrá que pese a sus quejas, el sujeto siempre es feliz, siempre goza. ${ }^{20}$

Si se piensa lo anterior aplicado al cuerpo, se trataría de que el registro de lo simbólico no es capaz de recoger todo aquello que lo precede: hay un agujero que el sujeto quiere tapar inútilmente con la palabra o con el deseo, pero que siempre está ya ahí funcionando en un nivel inferior. En el paso del organismo al cuerpo hay algo que se pierde y queda más allá -o más acá-, «éxtimo», al sujeto. ${ }^{21}$ La relación del sujeto con su cuerpo desde el punto de vista de lo real/ imposible es una relación de pérdida o, como mínimo, de desencuentro con su goce, puesto que la única herramienta que tiene para acceder a él -el lenguajeno es todo lo apropiada que debiera ser.

La recuperación parcial del goce de este cuerpo desertificado por la acción del lenguaje se produce tan solo en ciertas zonas, que son las zonas erógenas del cuerpo. El sujeto no se relaciona con las partes 'naturales' de su cuerpo sino que este está organizado en una serie de zonas erógenas que actúan como condensadoras de goce. Cualquier objeto puede ser objeto de la pulsión, que no es más que un circuito que da vueltas en torno a un objeto sin llegar nunca a captarlo por completo. El objeto $a$ no será solo objeto-causa de deseo sino

aquello que aquí se aparece, y que yo no lo articulo como un forzamiento [forçage: tratamiento para que las plantas crezcan fueran de sus etapas normales de crecimiento] [...] Lo que el análisis muestra si muestra alguna cosa [...], es precisamente esto, que no se transgrede nada. Deslizarse no es transgredir. Ver una puerta entreabierta, no es franquearla. Tendremos la ocasión de reencontrar esto que yo estoy introduciendo - no se trata aquí de transgresión, sino más bien de una irrupción, caída en el campo de alguna cosa que es del orden del goce - un excedente [boni]» (Lacan 1969-70, pp. 18-19).

20 «¿Dónde está en todo esto aquello que hace alegría? Por todas partes. El sujeto siempre es feliz» (Lacan 1974, p. 526).

$21 \ll[. .$.$] lo que describimos como ese lugar central, esa exterioridad íntima, esa extimidad,$ que es la Cosa» (Lacan 1959-60, p. 167). En los Escritos lo explica de la siguiente forma: «La heteronomía radical cuyo descubrimiento por parte de Freud ha mostrado la hiancia en el ser humano, no puede ser encubierta sin convertir a todo lo que se emplee para tal fin en una deshonestidad radical. ¿Cuál es entonces este otro al que estoy más unido que a mí mismo, porque en el seno más asentado de mi identidad conmigo mismo, es él el que me mueve?» (Lacan 1966, p. 524). 
también objeto en torno al que gira la pulsión del sujeto, ${ }^{22}$ pudiendo adoptar paradigmáticamente, aunque no exclusivamente, cuatro formas, todas ellas situadas sobre el cuerpo: mirada, voz, hez o pecho materno. En realidad Lacan terminará afirmando que la función ejercida por los objetos correspondientes a estas zonas -anal, escópico o de la mirada, de la succión o pecho, de la vozpuede ser desenvuelta por cualquier otro objeto. Frente al cuerpo simbólico que era un cuerpo desertificado del goce, Lacan relaciona ahora goce y significante, e intenta explicar cómo es posible recuperar algo de ese goce del organismo que el lenguaje barra y deja de lado. Es imposible obtener el goce de manera masiva, total; pero de manera fragmentaria, como objeto de una pulsión que da vueltas en torno a él, el sujeto tiene algún acceso a él. El cuerpo simbólico, el cuerpo situado en el campo del lenguaje, el cuerpo del Otro está agujereado en ciertas partes por los objetos $a$, que sin dejar de ser en una de sus caras elementos simbólicos, meros huecos o formas lógicas, son en la otra objetos causa de goce. Es tan solo gracias a los objetos a como se puede tener alguna experiencia del cuerpo.

Hacia finales de la década de los 70, Lacan propone una distinción entre dos tipos de goce motivado, al igual que lo estuviera Freud en Más allá del principio del placer, por el hecho de que si bien una parte del goce es puesta en palabras -con mayor o menor dificultad- otra permanece como una especie de resto indescifrable. Esto lo lleva a proponer que existe un goce fálico y un goce Otro. El goce fálico es el goce del falo; falo no en el sentido sexual sino en el sentido lacaniano de un significante destacado que ordena el discurso y el deseo del sujeto. Significantes que pertenecen a ese conjunto más o menos ordenado llamado Otro; si a este Otro le falta siempre algo y, por lo tanto, es siempre inconsistente, para que el lenguaje funcione es necesario algo que venga al lugar de esta falta y permita así que haya orden y significación. El falo es una especie de clave interpretativa que permite darle sentido al todo. Por lo tanto, el goce fálico, compartido por mujeres y hombres, tiene que ver con el sentido, con la creencia de que algo puede ser comprendido y explicado. El goce fálico sería aquella parte del goce que responde a la palabra y sobre el que, en consecuencia, se puede operar mediante el lenguaje; es el goce que se ofrece a interpretación y desciframiento. Se trata de un goce autoerótico, diferente en cada sujeto y que no permite que exista un contacto entre ellos. En realidad, pese a su nombre, y pese a ser el goce puesto en juego en el terreno de lo sexual, es más bien un goce autista que impide la relación. Este goce fálico aún siendo un

22 «Comprenda que el objeto del deseo es la causa del deseo y este objeto causa del deseo es el objeto de la pulsión, es decir, el objeto en torno del cual gira la pulsión» (Lacan 1964, p. 251). 
goce del órgano no es un goce que tenga que ver con el cuerpo. ${ }^{23}$ Esto insiste todavía más en el hecho de que se debe distinguir entre el cuerpo organizado en órganos -sobre el que el psicoanálisis no pretende realizar ninguna aportación innovadora- y el cuerpo como gozado.

Además de este goce fálico compartido por la posición masculina y por la posición femenina, esta última tiene acceso a un goce Otro exclusivamente femenino. ${ }^{24}$ Se trata de un goce que se aparece como un suplemento independiente del goce fálico y que está como a la deriva, inefable, enigmático. Lacan lo ilustra con el éxtasis místico de la Santa Teresa esculpida por Bernini que aparece en la portada de la edición francesa del seminario $\mathrm{XX} .{ }^{25} \mathrm{El}$ místico, en contacto con lo divino, sufre y disfruta al mismo tiempo sin límite: no puede decir nada de él, pero siente un goce enigmático en su cuerpo. En la medida en que la posición masculina y la posición femenina no vienen determinadas por la anatomía, no se prejuzga aquí que los varones no tengan acceso a sentir el goce Otro en su cuerpo. Se trata tan solo de dos lógicas que se distinguen para entender como se vive el propio cuerpo

\section{LA CONSISTENCIA DEL CUERPO EN EL ÚLTIMO LACAN: RETORNO A LO IMAGINARIO.}

En el capítulo X del seminario 23, Lacan se detiene en un pasaje del Retrato del artista adolescente donde Joye narra como el protagonista es golpeado por algunos compañeros de algunos compañeros y confiesa no haber sentido ni siquiera odio hacia ellos. Stephen sería capaz de separarse de su cuerpo como si fuese la piel de una fruta o una cáscara. ${ }^{26}$ Más allá de lo anómalo de la situación -puesto que lo normal es que el sujeto esté profundamente unido

23 «El goce, en la medida en que es sexual, es fálico, lo que significa que no se relaciona con el Otro en cuanto tal» (Lacan 1971, p. 14).

24 Este goce Otro «subsiste siempre en ella, distinto y paralelo del que obtiene por ser la mujer del hombre, aquel que se satisface del goce del hombre» (Lacan 1968-69, p. 386)

25 L'Estasi di Santa Teresa o Santa Teresa in estasi o Transverberazione di santa Teresa (1647-1651), escultura de Gian Lorenzo Bernini situada en la Iglesia de Santa María de la Victoria en Roma.

26 Joyce emplea esta metáfora en dos ocasiones. Una primera en la escena que cita Lacan: «Y aun aquella noche, al regresar vacilante hacia casa a lo largo del camino de Jone, había sentido que había una fuerza oculta que le iba quitando la capa de odio acumulado en un momento con la misma facilidad con la que se desprende la suave piel de un fruto maduro» (Joyce 1916, p. 92-93). Y otra posterior donde Joyce habla de sus sentimientos ante los dogmas cristianos: «A menudo había sentido un breve acceso de cólera, pero nunca había sido capaz de conservar su resentimiento largo rato, sino que había sentido que se iba desvaneciendo en seguida como una cáscara o una piel que se desprendiera con toda suavidad de su propio cuerpo» (Joyce 1916, p. 171). 
a su cuerpo y sufra en él los afectos que lo afecten-, a Lacan le sirve Joyce para mostrar como el cuerpo no es un organismo formado por órganos sino únicamente un cierto envoltorio, una figura, un continente o una línea que los rodea. ${ }^{27}$ En el protagonista del relato, que Lacan identifica con Joyce, el cuerpo no funciona, la consistencia que debería proporcionar está ausente y por ello es incapaz de sentir en su cuerpo los afectos de cólera que debería manifestar ante la afrenta recibida. Por otro parte, no se trata de que el cuerpo sea algo que se le escape únicamente a Joyce, sino que en realidad le pasa a cualquier sujeto. El ser-parlante, así denomina a los sujetos en su última época Lacan, considera que su cuerpo le pertenece y le obedece, pero en realidad él está siempre siempre escapándosele un poco, yendo por libre incontroladamente. Evidentemente no se trata de la falta de control que tenga el sujeto sobre su cuerpo orgánico -enfermedades, dolores, etc.- sino del hecho de que el cuerpo tiene un estilo propio independiente de la voluntad del sujeto. Su consistencia solo puede mantenerse de manera frágil y a base de creer en ella, de imaginarse la posesión del propio del cuerpo, de adorarlo, siendo en realidad una 'consistencia mental'. ${ }^{28}$

El sujeto no es, en definitiva, su cuerpo, sino que este está separado de él. Como mucho se podría decir que el sujeto tiene un cuerpo, pero aun así nunca se trataría de un tener como el que funciona cuando se dice «tengo un objeto»; el cuerpo no es un objeto que se puede usar y del que se puede disponer cuando se quiera. ${ }^{29}$ En comparación con el Inconsciente simbólico, con el Otro, con el lenguaje, con el sentido, el cuerpo es más bien del orden del malentendido, de un malentendido que no se puede leer, de algo que tiene la presencia definitiva de una cosa escrita pero que no puede terminar de descifrarse. ${ }^{30}$ El cuerpo es una

27 «QQué quiere decir la consistencia? Quiere decir lo que mantiene junto, y por eso aquí se la simboliza con la superficie. En efecto, pobres de nosotros, solo tenemos idea de consistencia por lo que constituye una bolsa o un trapo. Esta es la primera idea que tenemos al respecto. Incluso al cuerpo lo sentimos como piel que retiene en su bolsa un montón de órganos. En otras palabras, esta consistencia deja ver el hilo de la trama. Pero la capacidad de abstracción imaginativa es tan débil que excluye el nudo de este hilo - que se muestra como residuo de la consistencia» (Lacan 1975-76, p. 63).

28 «El amor propio es el principio de la imaginación. El parlêtre adora su cuerpo porque cree que lo tiene. En realidad, no lo tiene, pero su cuerpo es su única consistencia - consistencia mental, por supuesto, porque su cuerpo a cada rato levanta campamento» (Lacan 1975-76,p 64).

29 «Es preciso que ustedes capten lo que les he dicho de las relaciones del ser humano con su cuerpo, y que depende enteramente de que el ser humano dice que él tiene el cuerpo, su cuerpo. Ya decir 'su' es decir que lo posee, como un mueble por supuesto» (Lacan 1975-76, p. 151).

30 «Finalmente, es de todos modos del orden de lo escrito. En muchos casos no sabemos leerlo. Tendría que decir aquí algo que introdujese la noción de escrito. Todo sucede como si estuviese escrito en el cuerpo, algo que nos es dado como un enigma. No es para nada sorprendente que tengamos como analistas esa sensación» (Miller 1999, p. 137). 
especie de sin-sentido encarnado, por lo que antes que de interpretar y descifrar frontalmente sus síntomas -algo imposible-, en el último Lacan la propuesta es intentar imaginarizar, aplacar, ordenar, poner sentido sobre eso imposible que en el fondo será siempre salvaje y sin ley.

\section{CONCLUSIÓN: EL CUERPO MÁS ALLÁ DEL ORGANISMO.}

La industria farmacéutica y la ideología neurocientífica y genética que la acompaña están librando una batalla, con elevados intereses económicos de por medio, para imponer el dogma de que todo lo psíquico se puede reducir al ámbito de lo cerebral; el dogma de que los trastornos mentales son enfermedades orgánicas del cerebro constituye el pilar sobre el que se alza esta nueva religión que, tras haber conquistado las facultades de psiquiatría y psicología comienza a ganar adeptos también en las facultades de filosofía. ${ }^{31}$ La neurociencia y la industria farmacéutica parecen haberse puesto de acuerdo para hipermedicar a la población, comenzando por los casos infantiles de supuesta hiperactividad que acabarán desarrollando una adicción inútil de por vida a los derivados anfetamínicos. Al reducir el individuo, la persona y el sujeto a un organismo pensado como una mera extensión material, la medicina le niega al sujeto toda responsabilidad en aquello que le sucede a su cuerpo y promueve una dessubjetivación generalizada. Los problemas del cuerpo son pensados como algo puramente negativo que hay que eliminar de la forma más rápida posible, excluyendo de raíz toda pregunta acerca de la implicación del sujeto en su malestar. Una vez abandonado el concepto de la antigua alma y de las pasiones a ella asociada, por ser excesivamente espiritualista, la teorización filosófica del cuerpo debería ejercer toda la resistencia intelectual posible al proponer la centralidad del cuerpo y defender una subjetividad corporeizada, aunque, como ya se ha dicho, en una línea muy diferente a la de la filosofía de la mente contemporánea. El psicoanálisis lacaniano ofrece argumentos sólidos para este debate intelectual al sostener que los malestares subjetivos son malestares de un cuerpo y que el cuerpo no es simplemente un conjunto anatómico de órganos diseccionables. En la medida en que ninguno de los enfoques de Lacan viene a negar lo dicho en épocas anteriores de su pensamiento, sino a desarrollarlo, se ha visto como el cuerpo es: la imagen que permite la aparición de una identidad subjetiva, el lugar donde se inscriben los objetos-causa del deseo, portador de cierto imposible

31 Sirvan de ejemplos las siguientes convocatorias: «I Jornada internacional sobre neuroestética. El entendimiento cerebral de la belleza. 24 y 25 de abril del 2014. Instituto de salud Carlos III. Madrid». «II Congreso Internacional de Bioética, titulado «Bioética, Neuroética, Libertad y Justicia», Valencia (España), del 12 al 14 de noviembre de 2012». «Neurometafísica. Las consecuencias epistemológicas de las neurociencias». 11 de Noviembre de 2013. Campus San Joaquín. Universidad Católica de Chile». 
resistente a la voluntad del sujeto y, finalmente, y en una recuperación parcial de su primer enfoque, garante de la consistencia del sujeto.

\section{REFERENCIAS BIBLIOGRÁFICAS:}

DELEUZE, G. \& GUATTARI, F. 1980: Capitalismo y esquizofrenia. Mil mesetas. Valencia: Pretextos, 2000.

FOGLIA, L. \& WILSON, R. 2015: «Embodied Cognition» en The Stanford Enciclopedy of Philosophy (Winter 2012 Edition), Edward N. Zalta (ed.), URL = http://plato. stanford.edu/entries/embodied-cognition/.

FREUD, S. 1925: «El sepultamiento del complejo de Edipo». En S. Freud, Obras completas, vol. XIX. Buenos Aires: Amorrortu, 2000, pp. 177-187.

FREUD, S. 1911: «Puntualizaciones psicoanalíticas sobre un caso de paranoia (Dementia paranoides) descrito autobiográficamente». En S. Freud, Obras completas, vol. XII. Buenos Aires: Amorrortu, 1991, pp. 1-76.

FREUD, S. 1920: «Más allá del principio del placer»en Sigmund Freud, Obras Completas. XVIII. Buenos Aires: Amorrortu, 1996.

HEIDEGGER, M. 1950: La cosa. En M. Heidegger, Conferencias y artículos. Barcelona: ediciones del Serbal, 2001.

JOYCE, J. 1916: Retrato del artista adolescente. Madrid: Alianza editorial, 2007.

LACAN, J. 1932: De la Psicosis Paranoia en sus relaciones con la personalidad: seguido de Primeros escritos sobre la paranoia. Madrid: Editorial SGGI, 1975.

LACAN, J. 1937: «Les complexes familiaux dans la formation de l'individu». En J. Lacan, Autres Écrits. París: Seuil, 2012, pp. 23-84.

-_, 1953-54: El seminario 2: el yo en la teoría de Freud y en la técnica psicoanalítica. Buenos Aires: Paidós, 2001.

—-, 1959-60: El seminario 7: la ética del psicoanálisis. Buenos Aires: Paidós, 1990.

- - 1964: El seminario 11: los cuatro conceptos fundamentales del psicoanálisis. Buenos Aires: Paidós, 2006.

—-, 1966: «Observación sobre el informe de Daniel Lagache: 'Psicoanálisis y estructura de la personalidad'». En J. Lacan, Escritos. Buenos Aires: Siglo XXI, 2005, pp. 627-664.

- - 1966b: «La instancia de la letra en el inconsciente o la razón desde Freud». En J. Lacan, Escritos. Buenos Aries: Siglo XXI, 2005, pp. 461-507.

—-, 1966c: «Psicoanálisis y medicina». En J. Lacan, Intervenciones y textos. Buenos Aires: Manantial, 1985. 
——, 1966-77: El seminario 14. La lógica del fantasma. Inédito. Versión digital en Internet. http://www.bibliopsi.org/docs/lacan/17\%20Seminario\%2014.pdf.

—-, 1968-69: El seminario 16. De un Otro al otro. Buenos Aires: Paidós, 2008.

—-, 1969-70: El seminario 17. El reverso del psicoanálisis. Buenos Aires: Paidós, 1991.

—-, 1974-75: El seminario. Libro XXII. RSI. Inédito. Versión digital en Internet. http:// www.bibliopsi.org/docs/lacan/27\%20Seminario\%2022.pdf.

—-, 1974: «Telévision» en Jacques Lacan. Autres Écrits, Paris: Seuil, 2005, pp. 509-546.

_-, 1975-76: El seminario 23: el sinthome. Buenos Aires: Paidós, 2006.

——, 1977: Psicoanálisis. Radiofonía \& Televisión. Barcelona: Anagrama, 1977.

MILLER, J-A. 1999: Intervenciones y Textos 2. Buenos Aires: Manantial.

_-, 2002: Biología lacaniana y acontecimiento del cuerpo. Buenos Aires: Diva.

_-, 2013: El ultimísimo Lacan, Buenos Aires: Paidós.

Francisco Conde Soto es profesor interino por vacante a tiempo completo en el Departamento de Filosofía y Antropología de la Universidad de Santiago de Compostela.

Líneas de Investigación:

Relación entre psicoanálisis y marxismo.

Teorías de la subjetividad posestructuralistas, principalmente Deleuze y Lacan.

Publicaciones recientes:

«El 'Kant con Sade' de Jacques Lacan: renuncia al deseo y sadismo en el imperativo categórico kantiano» en Anales del Seminario de Historia de la Filosofía, vol. 34, núm. 2, 2017, pág. 85-106)

«Cuerpo y Feminidad : 'Goce Otro' de Jacques Lacan y 'Devenir-Mujer' en Deleuze y Guattari» en Trans/Form/Ação. Revista de Filosofia da UNESP, vol. 39, núm. 4, 2016, pág. 85-106.

Correo electrónico:

francisco.conde@usc.es 
